\title{
Gobierno corporativo, financiamiento y género: un estudio de las pymes emisoras de títulos en los mercados de valores argentinos
}

\author{
Corporate governance, financing and gender: A study of SMEs \\ from Argentinean Securities Markets
}

\author{
Anahí Briozzo $^{\mathrm{a}, *}$, Diana Albanese ${ }^{\mathrm{b}}$ y Diego Santolíquido ${ }^{\mathrm{b}}$ \\ a Departamento de Ciencias de la Administración, IIESS, Universidad Nacional del Sur (UNS)-CONICET, Bahía \\ Blanca, Argentina \\ b Departamento de Ciencias de la Administración, Universidad Nacional del Sur (UNS), Bahía Blanca, Argentina
} Recibido el 27 de noviembre de 2014; aceptado el 30 de junio de 2016

Disponible en Internet el 7 de febrero de 2017

\section{Resumen}

El objetivo de este trabajo es estudiar el gobierno corporativo (GC) y el financiamiento en pequeñas y medianas empresas (pymes) desde una perspectiva de género. En particular, se analiza la participación de mujeres en la propiedad, administración (directorio y alta gerencia) y auditoría externa en las pymes que participan de los mercados de valores argentinos. Los resultados muestran que existen relaciones significativas entre la participación de mujeres en la propiedad y la auditoría externa, y las decisiones de financiamiento. Sin embargo, no se encuentra relación entre la participación de mujeres en los distintos niveles del GC. (C) 2017 Universidad Nacional Autónoma de México, Facultad de Contaduría y Administración. Este es un artículo Open Access bajo la licencia CC BY-NC-ND (http://creativecommons.org/licenses/by-nc-nd/4.0/).

Códigos JEL: G34; G32; J16; M42

Palabras clave: Gobierno corporativo; Género; Pequeñas y medianas empresas; Financiamiento

\section{Abstract}

The aim of this work is to study corporate governance (CG) in small and medium enterprises (SMEs) from a gender perspective. In particular, we study the participation of women in ownership, management (board

\footnotetext{
* Autor para correspondencia.

Correo electrónico: abriozzo@uns.edu.ar (A. Briozzo).

La revisión por pares es responsabilidad de la Universidad Nacional Autónoma de México.
} 
and senior management) and external audit in SMEs participating in the Argentinean Securities Market. The results show that participation of women in ownership and in external audit has a significant relationship with financing decisions. However, we do not find evidence of relations among different levels of GC and gender.

(C) 2017 Universidad Nacional Autónoma de México, Facultad de Contaduría y Administración. This is an open access article under the CC BY-NC-ND license (http://creativecommons.org/licenses/by-nc-nd/4.0/).

JEL classification: G34; G32; J16; M42

Keywords: Corporate governance; Gender; Small and medium enterprises; Financing

\section{Introducción}

Según los datos del Banco Mundial ${ }^{1}$, en América Latina y el Caribe un 40.2\% de las empresas tienen al menos un propietario mujer, y el $21.1 \%$ de las firmas son dirigidas por una mujer. En la Argentina la participación en la propiedad de las mujeres es del $38 \%$, mientras que rol femenino en la gerencia general es menor que el promedio de la región, rondando el 9.2\%. La relevancia de la participación de las mujeres en la propiedad y dirección de las empresas, unido a las características diferenciales de las pequeñas y medianas empresas (pymes), ha promovido que una rama de los estudios sobre este sector se haya abordado desde una perspectiva de género. Este trabajo se inserta en el marco de los estudios sobre financiamiento y género en pymes, considerando el rol de la mujer en la propiedad y administración de la empresa, y a la vez se suma una nueva arista al problema: el rol de los otros mecanismos de gobierno corporativo (GC), como son el directorio y el auditor externo.

El desarrollo de los mecanismos de GC se vuelve especialmente relevante en las empresas que buscan fondos a largo plazo en los mercados de capitales, un segmento de financiamiento donde la presencia de las pymes aún es escasa. Según indica el Instituto Argentino de Mercado de Capitales (2013), en la Argentina el financiamiento a largo plazo (obligaciones negociables y fideicomisos financieros) destinado a pymes a través del mercado de capitales doméstico en 2013 fue del $14.6 \%$ de los fondos captados por el sector pyme, y del $0.9 \%$ del financiamiento empresario total. En pos de incentivar la participación de las pymes en el mercado de capitales, en la Argentina se han desarrollado regímenes especiales que facilitan los procesos y disminuyen los costos de la emisión de títulos para estas empresas (Resolución General de la Comisión Nacional de Valores [CNV] N. ${ }^{\circ} 336 / 99$, Normas CNV 2013). En particular, con respecto a los aspectos del GC, las pymes están exceptuadas de constituir un órgano colegiado de fiscalización y un Comité de Auditoría, y no están obligadas a presentar el Código de Gobierno Societario. Esto implica que la información sobre GC de estas empresas esté diseminada en los distintos documentos de acceso público que las mismas presentan a la $\mathrm{CNV}$, como balances, información de hechos relevantes y prospectos de emisión, entre otros.

En función de estos antecedentes, el objetivo de este trabajo es estudiar el GC y el financiamiento en pymes desde una perspectiva de género. El trabajo se aborda desde un punto de vista descriptivo-correlacional, siendo la población bajo estudio las pymes emisoras de obligaciones negociables en los mercados de valores argentinos. Los resultados muestran que existe relación

\footnotetext{
1 Banco Mundial, Resultados de la World Enterprise Surveys, disponible en: http://espanol.enterprisesurveys. org/data/exploreeconomies/2010/argentina\#gender
} 
entre el financiamiento de las empresas y la participación de las mujeres en la propiedad y en la auditoría externa. Sin embargo, no se encuentra relación entre los distintos mecanismos de GC y la participación femenina en ellos.

Los aportes de este trabajo subyacen en las siguientes bases: a) se estudia la relación entre GC, género y financiamiento desde una óptica amplia de los mecanismos de GC, considerando propiedad, directorio, alta gerencia y auditor externo; b) se estudian pymes de un país en vías de desarrollo, cuando la mayor parte de los trabajos previos se han basado en países desarrollados, y c) se analiza una base de datos única conformada con información que, si bien es de acceso público, no se encuentra sistematizada.

El trabajo se encuentra estructurado de la siguiente forma: primero se presentan el marco teórico y los antecedentes empíricos, en segundo lugar se presenta la metodología, a continuación se analizan los resultados, y se finaliza con las conclusiones.

\section{Marco teórico y antecedentes empíricos}

Se presenta en esta sección el marco conceptual y los estudios previos. Primero en lo referente a GC y pymes en general, segundo lo referido a GC y la participación de mujeres, y a continuación se aborda la relación entre género y financiamiento. Cada una de esta subsecciones se acompaña con la presentación de las hipótesis. Por último, se desarrolla el contexto temporal y geográfico de la participación de mujeres en la gestión y propiedad de las empresas.

\section{Gobierno corporativo en pymes}

El GC puede definirse como «el conjunto de principios, políticas, procedimientos, estándares y normas que se utilizan para dirigir y controlar una entidad de cualquier naturaleza, tamaño o condición, de una forma ética, equitativa y responsable. Ese conjunto regula el diseño, integración y funcionamiento de los órganos de gobierno, reflejando las relaciones de poder entre accionistas, directorio y alta gerencia» (Casal, 2010, p. 1).

El marco conceptual clásico del GC parte de la teoría de la agencia (Jensen y Meckling, 1976). En un contexto de grandes empresas de capital abierto con propiedad atomizada, surgen conflictos entre accionistas y directivos, derivados de la separación de propiedad y control (problema principal-agente). Sin embargo, en países emergentes, la concentración de la propiedad, sumado a mecanismos de gobernanza externa más débiles, resulta en conflictos entre accionistas mayoritarios y minoritarios, lo cual se conoce como conflicto principal-principal (Young, Peng, Ahlstrom, Bruton y Jiang, 2008). El conflicto principal-principal deriva en la extracción de riqueza de los accionistas minoritarios en manos de los mayoritarios, mediante la designación de personal poco calificado en posiciones clave (amigos y/o familiares), la adquisición de servicios o productos por precios sobrevalorados $-\mathrm{o}$ venta de productos a precios subvalorados- a entidades controladas por el mismo grupo de accionistas y el desarrollo de estrategias que favorecen interesen personales, familiares o políticos.

Las buenas prácticas de GC actuarían mitigando las asimetrías de información, y por lo tanto reduciendo los costos de agencia, lo cual se traduciría en un mejor desempeño de la empresa. Diversos trabajos encuentran evidencia a favor de esta relación; para grandes empresas en mercados emergentes, ver Campos, Newell y Wilson (2002); Klapper y Love (2004); Garay y González (2008); Braga Alves y Shastri (2011), entre otros. 
La literatura empírica sobre GC en pymes se ha desarrollado mayoritariamente en mercados desarrollados. Abor y Adjasi (2007) indican que los mecanismos de GC en pymes a los que se les presta más atención son:

- Separación de alta gerencia y directorio, para asegurar el rol de control del directorio sobre la gerencia. Esto incluye evitar la dualidad del CEO.

- Mezcla adecuada de directores independientes e internos.

- Independencia del auditor externo para asegurar la integridad de los reportes financieros.

Resulta interesante mencionar que, si bien los mecanismos de GC podrían ser en principio similares a los de las grandes empresas, la evidencia empírica en pymes no es homogénea. Por ejemplo, Arosa, Iturralde y Maseda (2013) reportan que el tamaño del directorio y el porcentaje de directores externos inciden negativamente en el rendimiento de las pymes españolas. Estos resultados indicarían los efectos de la falta de coordinación, flexibilidad y comunicación en un directorio de gran tamaño.

Brunninge, Nordqvist y Wiklund (2007) encuentran que en las pymes suecas con mayor concentración de la propiedad la tendencia al cambio estratégico es menor, lo cual estaría asociado con la aversión al riesgo y la resistencia al cambio. La presencia de directores externos y el número de miembros de la alta gerencia tienen un efecto positivo sobre los cambios estratégicos, y estos dos mecanismos actúan en cierto grado como sustitutos entre sí.

Dasilas y Papasyriopoulos (2015) estudian pymes y grandes empresas griegas de capital abierto, y encuentran que el tamaño del directorio tiene una relación negativa con la deuda a corto plazo, si bien esta relación es más débil para las empresas más pequeñas. Este resultado estaría explicado por la mayor participación de accionistas en los directorios de las empresas de menor tamaño, lo cual disminuiría las asimetrías de información (desde un enfoque de problema principal-agente).

Para la Argentina, Santolíquido, Briozzo y Albanese (2014) estudian la relación entre GC, edad y formación de los integrantes de los diferentes niveles organizacionales, y financiamiento y desempeño de las empresas. El tamaño del directorio incide negativamente en el plazo del endeudamiento. Se observa que para la variable edad del presidente del directorio, así como para el promedio de edad de los integrantes del directorio, hay una relación negativa con el ratio de Pasivo Total a Ventas. Un directorio con mayor formación académica tiende a endeudarse a mayor plazo, y también se asocia con mejor desempeño. Con respecto a la dualidad del CEO, en la mitad de las empresas en que el presidente del directorio tiene título en ciencias económicas, este es también el gerente general, mientras que este porcentaje baja al 10\% de las empresas cuyo presidente del directorio no tiene dicha formación.

\section{Género y gobierno corporativo}

Dentro de los mecanismos de GC que surgen para evitar que los agentes manipulen los resultados contables, utilizando diferentes principios y métodos contables, encontramos las figuras de los directores y los auditores externos. Nos interesa analizar la situación actual de estos mecanismos desde la perspectiva de género, es decir, evaluando el rol que desempeña la mujer en ellos.

El interés sobre este tema se remonta a Daily, Certo y Dalton (1999), quienes analizan cómo avanzó la participación femenina en los principales puestos gerenciales y directivos en la década de los noventa. A partir de este estudio, se desprenden una serie de investigaciones que estudian la temática desde diferentes puntos de vista y con objetivos diversos. Francoeur, Labelle y SinclairDesgagné (2008) intentan determinar cómo afecta al desempeño de la firma el hecho de tener 
mujeres en el directorio y gerencias de primera línea, desde la perspectiva de los stakeholders ${ }^{2}$ (partes interesadas). Encuentran que las firmas en las que las mujeres ocupan puestos gerenciales tienen mejor desempeño, pero que la mayor cantidad de mujeres en el directorio no asegura un mayor valor de mercado. Consistentemente con esto, Farrell y Hersch (2005) no encuentran relación positiva entre la diversidad genérica del directorio y el valor de mercado, lo cual les lleva a concluir que la inclusión de mujeres en puestos directivos se debe a otros factores (presiones externas, mejora de imagen, objetivos de la compañía, etc.).

Para saber cómo puede mejorar el desempeño de la firma a partir de la inclusión de un mayor porcentaje femenino en el directorio, es importante considerar cómo responden los inversores a este fenómeno. En este sentido, Kang, Ding y Charoenwong (2010) prueban que, en el caso de Singapur, los inversores responden positivamente a la adición de mujeres al directorio, y le atribuyen los resultados a diferentes razones: a que la comunidad de ese país ve favorablemente que la mujer tenga un estatus alto en la sociedad y ocupe un rol importante en la toma de decisiones, a que los empresarios líderes profesan los beneficios de los grupos diversificados, y a que estos últimos tienen sus desventajas (aumenta el tiempo necesario para la toma de decisiones y el consenso), pero que se dan principalmente en grupos altamente diversificados (no es el caso en Singapur).

López Vergara, Gómez-Betancourt y Betancourt Ramírez (2011) estudian los factores que influyen en la participación de mujeres en órganos de GC en empresas familiares en Colombia, y concluyen que son motivos relevantes la conservación del patrimonio, el crecimiento profesional, la preservación de la unidad de la familia, la contribución al desarrollo de la firma y la generación de espacios de comunicación familiar.

Asimismo, se debe tomar en cuenta cómo se da esta inclusión, la que puede provenir de diversas fuentes: presiones externas, políticas explícitas de la compañía, y para mejorar la imagen (Farrell y Hersch, 2005). Estos autores buscan explicar el motivo que puede causar la incorporación de una mujer al directorio, tomando en cuenta si se debe a un reemplazo por retiro de uno de sus miembros, y si el que parte es hombre o mujer, e intentan modelar la probabilidad de incorporar a una mujer en reemplazo de un miembro o aumentando el número de directores. Los resultados de su análisis muestran que la probabilidad de incluir a una mujer en el directorio en un año determinado está negativamente relacionada con el porcentaje de mujeres existentes en el año anterior. Concluyen que el género influencia la selección del directivo. En función de la relación positiva esperada con respecto a la participación femenina en los distintos órganos de GC, se postulan las siguientes hipótesis:

Hipótesis 1 (H1). Existe una relación positiva entre la participación en la propiedad de mujeres y la participación femenina en el directorio.

Hipótesis 2 (H2). Existe una relación positiva entre la participación en la propiedad de mujeres y la participación femenina en la alta gerencia.

Hipótesis 3 (H3). Existe una relación positiva entre la participación en el directorio de mujeres y la participación femenina en la alta gerencia.

Otros estudios se enfocaron en cómo afecta el género en el otro mecanismo de GC; la auditoría externa (O’Donnell y Johnson (2001); Ittonen, Vähämaa y Vähämaa (2013)). Los mismos parten

\footnotetext{
${ }^{2}$ Se considera como partes interesadas a todas las personas e instituciones que tienen algún tipo de relación con la empresa (empleados, gobierno, proveedores, propietarios, acreedores, público en general, etc.). Según esta perspectiva, la inclusión de la mujer en puestos directivos se da por la presión ejercida por estos grupos.
} 
de la hipótesis de selectividad ${ }^{3}$, e intentan determinar si mujeres y hombres auditores muestran diferencias basadas en el género en la resolución de diferentes problemas, comprobando que las mujeres se desempeñan mejor en los problemas complejos (les toma menos tiempo que a los hombres), pero los masculinos son más eficientes en tareas simples. Los resultados no implican que un género tome mejores decisiones que el otro, sino que dan un enfoque diferente a su desempeño, en cuanto a tiempo y complejidad. En función de la relación positiva esperada con respecto a la participación femenina en los distintos órganos de GC, se postulan las siguientes hipótesis:

Hipótesis 4 (H4). Existe una relación positiva entre la participación en la propiedad de mujeres y la existencia de una mujer como auditora externa.

Hipótesis 5 (H5). Existe una relación positiva entre la participación en el directorio de mujeres y la existencia de una mujer como auditora externa.

Hipótesis 6 (H6). Existe una relación positiva entre la participación en la alta gerencia mujeres y la existencia de una mujer como auditora externa.

\section{Género y financiamiento en pymes}

El estudio del impacto del género en las decisiones de financiamiento de las pymes se ha centrado tradicionalmente en el rol del propietario-administrador. Siguiendo a Constantinidis, Cornet y Asandei (2006), en esta relación género-financiamiento pueden identificarse factores asociados por un lado con la demanda de fondos (el comportamiento de mujeres empresarias versus hombres empresarios en las decisiones de financiamiento), y por el otro con la oferta (el comportamiento de los proveedores de fondos frente a empresarios mujeres versus hombres).

Por el lado de los argumentos de demanda, surgen características diferenciales de las mujeres como tomadoras de decisiones (visión social del feminismo), como por ejemplo una mayor aversión al riesgo. En función de esto, mujeres y hombres difieren en términos de motivación para iniciar una empresa, habilidades para los negocios, nivel de confianza en sí mismos y prioridades en las tareas administrativas (Jonhsen y McMahon, 2005). Por lo tanto, las diferencias en el financiamiento existentes en empresas lideradas por hombres y mujeres responderían en parte a diferencias en las características de las empresas que estos dirigen, en particular en cuanto a sector, tamaño, antigüedad y estructura de propiedad (Constantinidis et al., 2006). Las mujeres generalmente inician negocios de tamaño pequeño, en sectores tradicionales de bajo crecimiento (principalmente servicios y comercio minorista), con estructuras de un único propietario. Esto explicaría el menor capital inicial invertido, y el hecho de que sean emprendimientos menos atractivos para los proveedores de fondos.

Por el lado de los argumentos de oferta, se indica que a igualdad de condiciones, las mujeres sufren mayores obstáculos que los hombres al buscar financiamiento externo (visión feminista liberal). Las predicciones de ambos enfoques (demanda y oferta) son similares en términos de resultados observados, es decir, un menor uso de financiamiento externo por parte de las mujeres empresarias. Carter, Shaw, Lam y Wilson (2007, p. 428) señalan que «la evidencia considerando género, emprendedorismo y crédito bancario sugiere que los perfiles de financiamiento son distintivamente diferentes, atribuible en gran parte (pero no todo) a diferencias estructurales».

\footnotetext{
${ }^{3}$ Plantea que hombres y mujeres siguen diferentes estrategias de procesamiento de información, por lo que los hombres son más proclives a seguir procesos más simplificados, mientras que las mujeres siguen procesos más complejos.
} 
En función tanto de los argumentos de oferta y demanda respecto al rol del propietarioadministrador, se plantean las siguientes hipótesis:

Hipótesis 7 (H7). La participación femenina en la propiedad está asociada con menor endeudamiento y a un menor plazo.

Hipótesis 8 (H8). La participación femenina en la alta gerencia está asociada con menor endeudamiento y a un menor plazo.

Con respecto al rol de otros mecanismos de GC en el endeudamiento, Mínguez-Vera y López-Martínez (2010) analizan este fenómeno en las pymes españolas y encuentran que la representación femenina en el directorio genera un efecto positivo en el desempeño (los efectos positivos de la diversificación de género sobrepasan los negativos). Confirman el supuesto de que las mujeres en el directorio, al ser más adversas al riesgo que los hombres, se inclinan por trabajar en empresas con menor ratio de deuda y menor probabilidad de quiebra. Con base a esto, se plantean las siguientes hipótesis:

Hipótesis 9 (H9). La participación femenina en el directorio está asociada con menor endeudamiento y a un menor plazo.

Por último, Ittonen et al. (2013) confirman que las diferencias en cuanto a aversión al riesgo, diligencia y conservadurismo entre género tienen influencia en la calidad de los reportes contables, siendo las mujeres auditoras las más precisas y eficientes en su producción. Esto implica mayor seguridad en el reporte financiero, lo cual puede estar asociado a mayor confianza para la toma de deuda. Es por esto que se espera que las empresas cuyos estados financieros son auditados por mujeres se manejen con mayores niveles de deuda y a más largo plazo. Por lo tanto, se formula la siguiente hipótesis:

Hipótesis 10 (H10). Las firmas auditadas por mujeres toman más deuda y a más largo plazo que las auditadas por hombres.

\section{Contexto temporal y geográfico}

Según datos de Grant Thornton International ${ }^{4}$, la participación de mujeres en cargos directivos en la Argentina se encuentra debajo del promedio mundial (19.4\% vs $22.2 \%$ para el periodo 2011-2015). Tal como se expone en la figura 1, la participación de mujeres en cargos directivos de empresas privadas y públicas en la Argentina fue del 16\% para el año 2015, coincidiendo con el año 2007 en el cual se inició la medición. Durante el período transcurrido entre los años mencionados se manifestaron incrementos en los porcentajes de participación, para disminuir posteriormente en el último año analizado. Un análisis similar se puede realizar sobre el promedio mundial de la ocupación femenina de cargos directivos y gerenciales. En cuanto a otros países de América Latina, se encuentra que la tendencia es coincidente con el promedio mensual en México, aunque el porcentaje participativo es superior a la Argentina. Un dato relevante es la

\footnotetext{
${ }^{4}$ Empresa que presta servicios de auditoría, consultoría y asesoría de negocios a empresas privadas y públicas, siendo Grant Thornton Argentina - como cada una de las firmas miembro y corresponsales — una firma autónoma. La mencionada firma emite el denominado International Business Report (IBR), elaborado mediante encuestas a empresas públicas y privadas. Los datos surgen de entrevistas a más de 5.000 directores ejecutivos, directores generales, presidentes y ejecutivos seniors.
} 


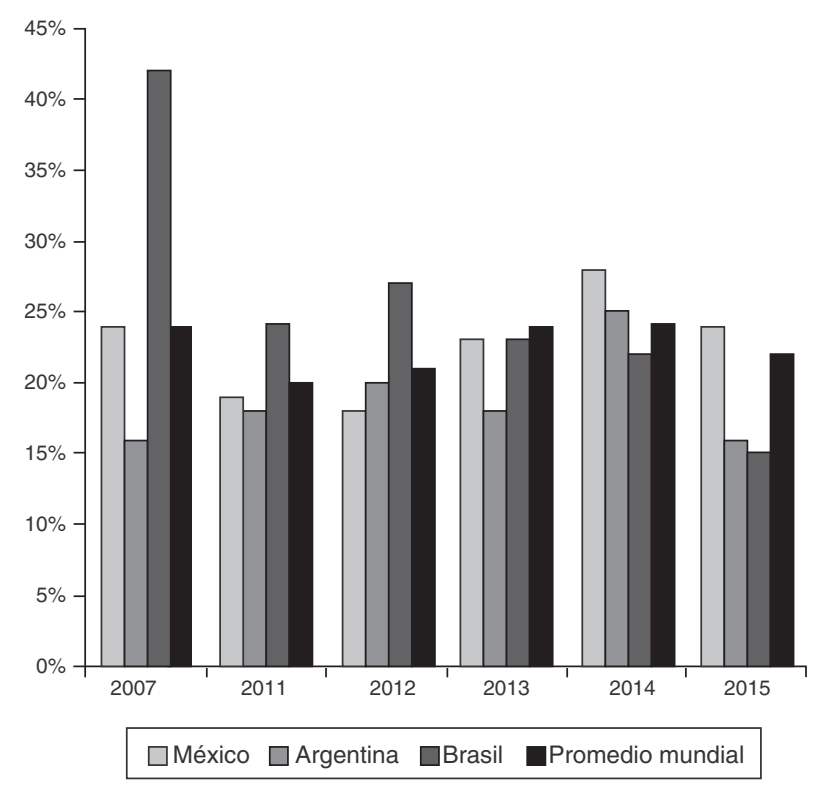

Figura 1. Evolución de la participación de mujeres en cargos directivos Fuente: Grant Thornton International (2015).

significativa disminución de la participación de la mujer en Brasil, que en el año 2007 era del 42\%, siendo en el 2015 un punto menos que en la Argentina.

Algunos de los motivos que explicarían la baja participación femenina se relacionan con la desaceleración económica de los países mencionados y a dificultades para las mujeres con hijos, no solo en cuanto al costo del cuidado de los niños sino a cuestiones complejas de planificación familiar. En la Argentina, los cargos directivos ocupados por mujeres son directoras generales, directoras de recursos humanos y, en menor medida, presidentes o vicepresidentes de empresas.

En los informes de Grant Thornton International se manifiesta un creciente apoyo al establecimiento de cuotas para que mayor cantidad de mujeres integren los consejos directivos. El mayor impulso está dado por la Unión Europea.

El Banco Mundial también genera información de la participación femenina en la propiedad y en altos puestos directivos, a través de su World Enterprise Surveys, que incluye empresas de más de cinco empleados. En la tabla 1 puede observarse que en la Argentina la participación de mujeres en la propiedad es en promedio similar al resto de América Latina, pero al discriminarse por tamaño de empresa, dicha participación disminuye en forma más marcada que para el resto de los países, en especial para las empresas de mayor tamaño. Asimismo, al analizar la participación femenina como principal directivo de la empresa, el promedio es notablemente inferior que para el resto de los países. En este aspecto, la participación de mujeres disminuye con el tamaño de la empresa para todos los países, pero lo hace en forma más marcada en la Argentina.

Con respecto a la normativa sobre participación femenina, no existen en la Argentina leyes que regulen la participación de las mujeres en el directorio de las empresas. En el ámbito de la política, rige desde el año 1991 la Ley Nacional 24.012, conocida como la ley de cupo femenino, que requiere que las listas partidarias para ocupar cargos electivos nacionales deben estar integradas, como mínimo, con un $30 \%$ de mujeres. Ha sido un avance importante no solo para el desarrollo de la mujer sino desde el punto de vista social para el tratamiento de temas como igualdad de género, 
Tabla 1

Participación femenina en la propiedad y en el principal cargo directivo, por tamaño de empresa

\begin{tabular}{llll}
\hline & Argentina & $\begin{array}{l}\text { Latinoamérica } \\
\text { y Caribe }\end{array}$ & Mundial \\
\hline Porcentaje de empresas con participación de mujeres en la propiedad & 38 & 39.80 & 33.90 \\
Pequeñas (5 a 19 empleados) & 46.90 & 41.20 & 33.70 \\
Medianas (20 a 99 empleados) & 30.40 & 39.50 & 33.90 \\
Grandes (más de 100 empleados) & 16.30 & 31.50 & 36.80 \\
Porcentaje de empresas con principal directivo mujer & 9.20 & 21.10 & 17.30 \\
Pequeñas (5 a 19 empleados) & 11.90 & 23.70 & 19.60 \\
Medianas (20 a 99 empleados) & 6.90 & 20 & 14.30 \\
Grandes (más de 100 empleados) & 3.60 & 10.60 & 10.20 \\
\hline
\end{tabular}

Fuente: Banco Mundial (2010).

defensa de derechos de la mujer y los niños, entre otros. No obstante la exitosa participación de la mujer a partir de la ley de cupo femenino, la misma no ha garantizado transformaciones profundas en cuanto a la igualdad de género.

\section{Metodología}

Esta sección presenta los detalles metodológicos del trabajo. Se define primero la población bajo estudio y las fuentes de datos. A continuación se muestran las herramientas estadísticas utilizadas en el análisis, y por último se detallan las definiciones de las variables.

\section{Datos}

El universo bajo estudio son las pymes emisoras de valores negociables desde enero de 2011 hasta abril de 2014 en los mercados de valores argentinos. Se consideran solo aquellas empresas cuya forma legal es sociedad anónima. La definición de pyme corresponde a la Resolución General 582/10 de la CNV (emisiones 2011-2012) y la Resolución 50/13 de la Secretaría de la Pequeña y Mediana Empresa y Desarrollo Regional (emisiones 2013 y 2014) ${ }^{5}$.

La población totaliza 33 pymes, de las cuales tres empresas son emisoras de acciones y el resto únicamente ha emitido obligaciones negociables. El listado completo de empresas se brinda en el Anexo, tabla A.2. Se utiliza la base de datos de Santolíquido et al. (2014), donde se estudian variables del GC, financiamiento y desempeño, evaluados diferencialmente según edad y formación de los integrantes de los diferentes niveles organizacionales. A diferencia de dicho trabajo, en el presente artículo el objetivo es analizar GC y financiamiento desde una perspectiva de género.

Según el Ministerio de la Industria de la República Argentina las pymes en el país son 603.000 empresas. Las 33 empresas que conforman la población bajo estudio, que son pymes que hacen oferta pública de sus títulos de deuda y/o capital, son una muy pequeña fracción de estas empresas. Sin embargo, son un conjunto de gran interés debido a que son empresas que han accedido al mercado de capitales, conforman un segmento de financiamiento que las políticas públicas nacionales han buscado desarrollar y fomentar para las pymes. Asimismo, por ser empresas bajo régimen de oferta pública, la información sobre ellas está disponible en forma pública y gratuita.

\footnotetext{
${ }^{5}$ Ver Anexo, tabla A.1, para mayor detalle sobre cómo se determina la condición de pyme.
} 
Bajo la regulación argentina, las pymes están exceptuadas de constituir un órgano colegiado de fiscalización y un Comité de Auditoría, y no están obligadas a presentar el Código de Gobierno Societario. Esto implica que la información sobre GC de estas empresas está diseminada en los distintos documentos de acceso público que las mismas presentan a la CNV, como balances, información de hechos relevantes, prospectos de emisión, entre otros ${ }^{6}$. Por lo tanto, para llevar a cabo este estudio fue necesario recabar y sistematizar toda esta información dispersa en una única base de datos. Las variables de GC corresponden a los prospectos de emisión, mientras que los datos financieros se miden en el momento de emisión y el último cierre de ejercicio. Teniendo en cuenta la información completa para todas las empresas, la muestra totaliza 22 firmas.

\section{Herramientas estadísticas}

El estudio realizado cuantitativo, de tipo descriptivo-correlacional. Para el análisis de relaciones entre variables se emplean los siguientes métodos:

- Análisis bivariado con variable dependiente cuantitativa. Dado el reducido tamaño de la población bajo estudio no se puede asumir normalidad de los datos, por lo que se emplea el coeficiente de correlación por rangos de Spearman para pares de variables cuantitativas (por ejemplo, porcentaje de mujeres accionistas y porcentaje de mujeres en la alta gerencia), y los test de Mann-Whitney y de Kruskal-Wallis para analizar una variable cuantitativa contra una categórica (por ejemplo, porcentaje de mujeres en el directorio y auditora mujer).

El coeficiente de correlación de Spearman (rho) es una medida de la asociación lineal entre dos variables aleatorias continuas. Se utilizan los rangos, números de orden, de cada grupo de sujetos y se comparan dichos rangos. El valor de rho oscila entre -1 (asociación perfecta negativa) y +1 , (asociación perfecta positiva), y se calcula como:

$$
r h o=1-\frac{6 \sum d_{i}^{2}}{N\left(N^{2}-1\right)}
$$

Donde: $\mathrm{d}_{\mathrm{i}}=$ diferencia entre los rangos $\left(\mathrm{x}_{\mathrm{i}}\right.$ menos $\left.\mathrm{y}_{\mathrm{i}}\right) ; \mathrm{N}=$ número de observaciones.

El test U de Mann-Whitney es un método no paramétrico que testea diferencias en una variable continua contra una variable nominal cuando esta tiene dos categorías. La hipótesis nula es que las medianas de las poblaciones de las que provienen las dos muestras son iguales. El test U se calcula como:

$$
U=n_{1} n_{2}+\frac{n_{2}\left(n_{2}+1\right)}{2}-\sum_{i=n_{1}+1}^{n_{2}} R_{i}
$$

Donde: $\mathrm{n}_{1}=$ tamaño de la muestra $1 ; \mathrm{n}_{2}=$ tamaño de la muestra $2 ; \mathrm{R}_{\mathrm{i}}=$ rango de la muestra $\mathrm{i}$.

La prueba de Kruskall-Wallis testea diferencias en una variable continua contra una variable nominal cuando esta tiene tres o más categorías. Es un test no paramétrico, ya que no requiere el supuesto de normalidad. Consiste en ordenar todas las observaciones de la muestra de mayor a menor, y asignar los rangos consecutivamente. Luego, se suman los rangos asignados a cada

\footnotetext{
${ }^{6}$ Información disponible en la sección de Información Financiera de la CNV, http://www.cnv.gob.ar/info Financiera.asp?Lang=0
} 
observación agrupándolos en grupos para cada uno de los grupos de estudio y se comparan las sumas obtenidas en cada uno de los grupos mediante un estadístico de contraste, evaluando su valor respecto a la ley de distribución de la Chi cuadrado con $\mathrm{k}-1$ grados de libertad, donde $\mathrm{k}$ indica el número de grupos que se están comparando. El estadístico de Kruskal-Wallis $(\mathrm{H})$ se calcula como:

$$
H=\frac{12}{N(N+1)} \sum_{i=1}^{k} \frac{R_{i}^{2}}{n_{i}}-3(N+1)
$$

Donde: $\mathrm{n}_{\mathrm{i}}(\mathrm{i}=1,2, \ldots, \mathrm{k})=$ el tamaño de cada una de las muestras para los $\mathrm{k}$ grupos de los datos. Cada uno de los $n_{i}$ debe ser como mínimo 5 para que el test sea válido; $R_{i}=$ la suma de los rangos del grupo i; $\mathrm{N}=$ número total de observaciones en todas las muestras.

- Análisis bivariado con variable dependiente categórica. Para testear la independencia entre dos variables categóricas se aplican los test de Pearson chi $^{2}$ y Fisher's exact. Dos variables categóricas (como principal accionista mujer y auditora mujer) se consideran independientes si todas las probabilidades conjuntas son iguales al producto de las probabilidades marginales. En este caso la hipótesis nula sería, por ejemplo, que el porcentaje de auditoras mujeres es igual cuando el principal accionista es hombre o cuando es mujer, la cual se rechaza si el valor de $\mathrm{p}$ es inferior al nivel crítico (por ejemplo, 5\%).

El test de Pearson chi ${ }^{2}$ se calcula como:

$$
\chi^{2}=\sum_{i=1}^{n} \frac{\left(O_{i}-E_{i}\right)^{2}}{E_{i}}=N \sum_{i=1}^{n} p i\left(\frac{o_{i} / N-p_{i}}{p_{i}}\right)^{2}
$$

Donde: $\chi^{2}=$ valor del estadístico; $O_{i}=$ número de observaciones del tipo i; $N=$ número total de observaciones; $E_{i}=N p_{i}=$ la frecuencia esperada del tipo i, derivada de la hipótesis nula de que la fracción de i en la población es $p_{i} ; n=$ número de celdas en la tabla.

El Fisher's exact se emplea cuando hay alguna celda en la tabla de contingencias con $\mathrm{n}<10$. Dadas dos variables $\mathrm{X}$ e $\mathrm{Y}$, con $\mathrm{m}$ y n estados observados respectivamente, se arma una matriz de dimensiones $\mathrm{m} \times \mathrm{n}$, donde los $\mathrm{a}_{\mathrm{ij}}$ representan el número de observaciones en donde $\mathrm{x}=\mathrm{i}$ y $\mathrm{y}=\mathrm{j}$. Se calcula la suma de las filas y la columnas, $\mathrm{R}_{\mathrm{i}} \mathrm{y} \mathrm{C}_{\mathrm{j}}$, respectivamente, y la suma total $N=\sum_{i} R_{i}=\sum_{j} C_{j}$. Luego se calcula la probabilidad condicional de obtener la matriz observada dada la suma particular de las filas y columnas, dado por:

$$
P=\frac{\left(R_{1} ! R_{2} ! \ldots R_{m} !\right)\left(C_{1} ! C_{2} ! \ldots C_{m n} !\right)}{N ! \prod_{i j} a_{i j} !}
$$

Que es una generalización multivariada de la función de probabilidad hipergeométrica.

Agrestri (2002) brinda mayor información sobre estos métodos.

Se estudiaron también las relaciones empleando métodos de análisis multivariado, pero la significatividad global de las diferentes regresiones, debido al reducido tamaño de la muestra, fue 
baja $^{7}$. A fines de detectar la posible presencia de relación espuria entre GC, género y financiamiento, se analizó también la relación con un grupo de variables de control, como se detalla en la sección siguiente.

\section{Variables}

En la tabla 2 se presenta la descripción operativa de las variables utilizadas en el análisis. El análisis estadístico de estas variables se realizó en dos etapas:

1. Análisis de variables de GC y género (porcentaje de mujeres accionistas, tenencia accionaria en mujeres, principal accionista es mujer, porcentaje de mujeres en el directorio, porcentaje de mujeres en la alta gerencia y auditor mujer) y las variables de financiamiento (nivel de endeudamiento y plazo).

2. Análisis de las variables de GC y género y un grupo de variables de control: el sector, la antigüedad, el tamaño (ventas), si la empresa es familiar, participación del principal accionista y porcentaje de directores externos. También se testeó la relación de las diversas variables de GC y género entre sí, a fines de detectar relaciones espurias en la etapa anterior.

\section{Resultados}

En primer lugar, en las tablas 3 y 4 se presentan las características de la población bajo estudio. Se destaca la baja participación de mujeres en la propiedad y administración de la empresa, siendo la auditoría externa el aspecto de GC con mayor participación femenina. La propiedad se encuentra fuertemente concentrada (media del 55\%), y el tamaño promedio del directorio es de 3 personas. En cuanto al financiamiento de las empresas, predomina el empleo de pasivos a corto plazo. Solo cuatro empresas poseen participación de capitales extranjeros en su propiedad.

En la tabla 5 se exhiben los resultados del análisis bivariado. Los resultados se presentan en tres bloques. En el primer panel se muestran las relaciones de las variables de GC y género entre sí. En el segundo panel se presentan los resultados de las variables de CG y género y las variables de financiamiento (plazo y fuente). Por último, el tercer panel presenta las relaciones de las variables de GC y género, y las variables control (antigüedad, sector, empresa familiar, tamaño, porcentaje de directores externos y participación del principal accionista)

- Propiedad y género. Al analizar la relación entre estas variables y el financiamiento (panel B), se encuentra que las firmas cuya principal accionista es mujer tienden a endeudarse más a corto plazo. En cambio, el porcentaje de mujeres accionistas se relaciona negativamente con el plazo, mientras que la concentración de tenencia accionaria en mujeres no tiene relación con el financiamiento.

Como es esperado, las tres variables de propiedad y género se encuentran relacionadas entre sí (panel A). El porcentaje de accionistas mujeres y la tenencia accionaria en mujeres presenta un rho de 0.7. Cuando la principal accionista es mujer, el $51 \%$ de los accionistas también lo

\footnotetext{
7 Se testearon distintos modelos econométricos: mínimos cuadrados ordinarios, modelo lineal generalizado y descomposición por cuantiles (quantil regression). En todos los casos se combinó como variable dependiente una medida del financiamiento y solo dos regresores, debido al reducido tamaño de la muestra. La elección de regresores consistió en incorporar una variable de gobierno corporativo y género y una variable control.
} 
Tabla 2

Definición operativa de las variables

\begin{tabular}{|c|c|}
\hline Variable & Definición \\
\hline \multicolumn{2}{|l|}{ GC y género } \\
\hline Principal accionista es mujer & $\begin{array}{l}\text { Toma valor «1» si el principal accionista de la firma es una mujer. } \\
\text { Variable binaria }\end{array}$ \\
\hline Porcentaje de mujeres accionistas & $\begin{array}{l}\text { Porcentaje de mujeres que son accionistas sobre el total de personas } \\
\text { físicas accionistas. Variable cuantitativa }\end{array}$ \\
\hline Propiedad en manos de mujeres & $\begin{array}{l}\text { Porcentaje del capital accionario en manos de mujeres. Variable } \\
\text { cuantitativa }\end{array}$ \\
\hline Porcentaje de mujeres en directorio & $\begin{array}{l}\text { Porcentaje que el número de mujeres directoras representa del total del } \\
\text { directorio. Variable cuantitativa }\end{array}$ \\
\hline Porcentaje de mujeres en alta gerencia & $\begin{array}{l}\text { Porcentaje que el número de mujeres gerentes representa del total de la } \\
\text { alta gerencia. Variable cuantitativa }\end{array}$ \\
\hline Auditor es mujer & $\begin{array}{l}\text { Toma valor «1» si los estados financieros son auditados por una mujer. } \\
\text { Se lo observa en el último cierre y en el cierre presentado en el } \\
\text { prospecto. Variable binaria }\end{array}$ \\
\hline \multicolumn{2}{|l|}{ Variables control } \\
\hline Empresa familiar & $\begin{array}{l}\text { Se considera empresa familiar si la propiedad está concentrada } \\
\text { totalmente o en su mayoría en personas con el mismo apellido } \\
\text { (o relación explícitamente establecida). Variable binaria }\end{array}$ \\
\hline Director externo & $\begin{array}{l}\text { Se considera director externo a aquel que no es propietario ni empleado } \\
\text { de la empresa. Se calcula la proporción de directores externos respecto } \\
\text { al total. Variable cuantitativa }\end{array}$ \\
\hline Antigüedad en oferta pública & $\begin{array}{l}\text { Años transcurridos desde la publicación del primer prospecto de } \\
\text { emisión de obligaciones negociables hasta la publicación del prospecto } \\
\text { de la emisión considerado en este estudio. Variable cuantitativa }\end{array}$ \\
\hline Antigüedad & $\begin{array}{l}\text { Años transcurridos desde la fecha de inicio de actividades hasta la } \\
\text { fecha del prospecto de emisión analizado. Variable cuantitativa }\end{array}$ \\
\hline Ventas & $\begin{array}{l}\text { Ventas del ejercicio, medido para el cierre de actividad de } 2013 . \\
\text { Variable cuantitativa }\end{array}$ \\
\hline Servicios & $\begin{array}{l}\text { Toma valor «1» si la empresa se desempeña en un sector de actividad } \\
\text { dentro del rubro servicios. Variable binaria }\end{array}$ \\
\hline \multicolumn{2}{|l|}{ Financiamiento } \\
\hline Pasivo Total sobre Patrimonio Neto & $\begin{array}{l}\text { Medido para el cierre de actividad de } 2013 \text { y del presentado en el } \\
\text { prospecto de emisión. Mide el ratio de financiamiento. Variable } \\
\text { cuantitativa }\end{array}$ \\
\hline Pasivo Corriente sobre Pasivo Total & $\begin{array}{l}\text { Medido para el cierre de actividad de } 2013 \text { y del presentado en el } \\
\text { prospecto de emisión. Refleja la proporción de deuda de corto plazo } \\
\text { sobre deuda total. Variable cuantitativa }\end{array}$ \\
\hline Pasivo Total a Ventas & $\begin{array}{l}\text { Medido para el cierre de actividad de } 2013 \text { y del presentado en el } \\
\text { prospecto de emisión. Permite relativizar los pasivos de la empresa con } \\
\text { una variable de flujo. Variable cuantitativa }\end{array}$ \\
\hline
\end{tabular}

Fuente: elaboración propia.

son, contra el $23 \%$ cuando es un hombre. A su vez, la tenencia accionaria en mujeres es del $53 \%$ cuando la principal accionista es mujer, contra el $6 \%$ cuando no lo es. Sin embargo, los resultados disímiles encontrados respecto al endeudamiento señalarían que las distintas formas de medir la participación de mujeres en la propiedad no son sustitutos perfectos entre sí.

En cuanto al análisis de variables control (panel C), se observa que la tenencia accionaria en mujeres tiene una relación positiva con el tamaño medido como ventas $(r h o=0.35)$. Como 
Tabla 3

Estadística descriptiva: variables cuantitativas

\begin{tabular}{|c|c|c|c|c|}
\hline Variable & Media & Desvío estándar & Min & Max \\
\hline \multicolumn{5}{|l|}{ Variables control } \\
\hline Antigüedad (años) & 17,8 & 13,1 & 0,96 & 55,18 \\
\hline Número de empleados (2013) & 129,9 & 190,4 & 8 & 871 \\
\hline Ventas (2013, en millones de US\$) & 24,69 & 5,6 & 13,26 & 36,03 \\
\hline Antigüedad en oferta pública (años) & 1,60 & 1,98 & 0,00 & 6,55 \\
\hline \multicolumn{5}{|l|}{ Variables de gobierno corporativo } \\
\hline Número de accionistas ${ }^{\mathrm{a}}$ & 4,16 & 2,37 & 2,00 & 10,00 \\
\hline Porcentaje de accionistas mujeres $^{\mathrm{a}}$ & $27,77 \%$ & $30,83 \%$ & $0 \%$ & $100 \%$ \\
\hline Participación del principal accionista ${ }^{a}$ & $55,46 \%$ & $27,56 \%$ & $20 \%$ & $99 \%$ \\
\hline Propiedad en manos de mujeres & $12,29 \%$ & $22,07 \%$ & $0 \%$ & $84,78 \%$ \\
\hline Número de personas en alta gerencia & 3,65 & 1,77 & 1,00 & 7,00 \\
\hline Porcentaje de mujeres en alta gerencia & $16,75 \%$ & $26,17 \%$ & $0 \%$ & $85,7 \%$ \\
\hline Número de miembros del directorio & 3,61 & 0,86 & 3,00 & 6,00 \\
\hline Porcentaje de mujeres en directorio & $13,98 \%$ & $18,96 \%$ & $0 \%$ & $66,67 \%$ \\
\hline Directores externos (porcentaje) & $34,52 \%$ & $35,64 \%$ & $0 \%$ & $100 \%$ \\
\hline \multicolumn{5}{|l|}{ Financiamiento } \\
\hline Pasivo corriente a Pasivo total (2013, PC/PT) & 0,81 & 0,19 & 0,33 & 1,00 \\
\hline Pasivo total a Patrimonio Neto (2013, PT/PN) & 4,27 & 7,11 & 0,84 & 40,45 \\
\hline Pasivo total a Ventas (2013, PT/V) & 2,30 & 6,15 & 0,09 & 28,68 \\
\hline
\end{tabular}

a Solo para empresas de capital cerrado.

Nota: valores en dólares americanos convertidos según dólar minorista, vendedor Banco Nación promedio para el año 2013 (US\$ = 5.467).

Fuente: elaboración propia.

el efecto esperado del tamaño de la empresa en el plazo de financiamiento es positivo, debido a las menores asimetrías de información, esta relación actuaría a lo sumo atenuando el efecto negativo observado en la relación principal accionista mujer y plazo de financiamiento, pero no ocasionaría una relación espuria. Otro efecto interesante es que la participación de mujeres en la propiedad implica una menor concentración de la misma: hay una relación negativa entre la participación del principal accionista y la tenencia accionaria en mujeres (rho $=-0.57$ ) y si el principal accionista es mujer (concentración promedio de 0.6 cuando el principal accionista es hombre, y de 0.25 cuando es mujer).

De las tres variables de propiedad y género estudiadas, consideramos la existencia de una accionista principal mujer como el indicador más fuerte de la influencia de la mujer en la toma de decisiones. La tenencia accionaria en mujeres y el porcentaje de mujeres accionistas, si bien son una medida de diversidad de género en la propiedad, pueden estar influidas por otros aspectos que también influyen en la toma de decisiones, como la concentración accionaria.

Tabla 4

Estadística descriptiva: variables categóricas

\begin{tabular}{llll}
\hline Variables control & $\%$ & Variables de GC & $\%$ \\
\hline Participación del sector servicios & 40.63 & Principal accionista mujer & 14.29 \\
Empresa familiar & 53.6 & Auditor mujer (2013) & 29.03 \\
\hline
\end{tabular}

Nota: Se indica participación de la característica presentada.

Fuente: elaboración propia. 
Tabla 5

Resultados del análisis bivariado

Panel A: GC y género

Relación positiva entre porcentaje de accionistas mujeres y la tenencia accionaria en mujeres ( $r h o=0.7, p=0.0002$ )

Relación positiva entre principal accionista mujer y porcentaje de accionistas mujeres: cuando la principal accionista es mujer, el $51 \%$ de los accionistas también lo son, contra el $23 \%$ cuando es un hombre $(\mathrm{p}=0.06)$ Relación positiva entre principal accionista mujer y tenencia accionaria en mujeres: la tenencia accionaria en mujeres es del $53 \%$ cuando la principal accionista es mujer, contra el $6 \%$ cuando no lo es $(\mathrm{p}=0.001)$

No hay relación entre porcentaje de mujeres accionistas, porcentaje de mujeres en el directorio y porcentaje de mujeres en la alta gerencia. Tampoco hay relación de estas variables con la presencia de una auditora mujer

Panel B: GC, género y financiamiento

\section{Propiedad y género}

Relación positiva entre principal accionista mujer y deuda a corto plazo: ratio PC/PT 91\%) cuando la principal accionista es mujer, $\mathrm{PC} / \mathrm{PT}=83 \%$ cuando no lo es $(\mathrm{p}=0.045)$. No tiene relación con el tipo de apalancamiento El porcentaje de mujeres accionistas se relaciona negativamente con el plazo de endeudamiento (rho $=-0.42$, $\mathrm{p}=0.054)$. No hay relación con el tipo de endeudamiento

No se encuentran relaciones significativas para la tenencia accionaria en mujeres y plazo y tipo de financiamiento Administración y género

La participación de mujeres en el directorio no se relaciona con las variables de financiamiento

La participación de mujeres en la alta gerencia no se relaciona con las variables de financiamiento

Auditor mujer

Relación positiva entre auditora mujer y endeudamiento total: $\mathrm{PT} / \mathrm{PN}=10.1$ cuando la auditora es mujer, $\mathrm{y}$

$\mathrm{PT} / \mathrm{PN}=2.54$ cuando no lo es $(\mathrm{p}=0.005)$. No hay relación con las restantes variables de endeudamiento

Panel C: GC, género y variables control

\section{Propiedad y género}

Relación positiva entre tenencia accionaria en mujeres y el tamaño de la empresa medido como ventas $($ rho $=0.35$, $\mathrm{p}=0.064)$

Relación negativa entre la participación del principal accionista y la tenencia accionaria en mujeres $($ rho $=-0.57$, $\mathrm{p}=0.001)$

Relación negativa entre la tenencia accionaria del principal accionista y si el principal accionista es mujer: concentración promedio de 0.6 cuando el principal accionista es hombre, y de 0.25 cuando es mujer $(\mathrm{p}=0.003)$ No se encuentran diferencias significativas para las variables empresa familiar, antigüedad, sector y directores externos

Administración y género

La participación de mujeres en el directorio es mayor en las empresas familiares $(22.7 \%$ vs $4.1 \%, p=0.015)$. No se encuentran diferencias significativas para las variables antigüedad, sector, tamaño, participación principal accionista y directores externos

El porcentaje de mujeres en la alta gerencia se relaciona positivamente con el porcentaje de directores externos $($ rho $=0.4, \mathrm{p}=0.073$ ). No hay relación con antigüedad, sector, tamaño, participación principal accionista y empresa familiar

Auditor y género

No hay relación entre la presencia de una mujer auditora y antigüedad, sector, tamaño, empresa familiar, participación principal accionista y directores externos

Nota: Los aspectos de propiedad y género se analizan solo para las empresas de capital cerrado.

Fuente: elaboración propia.

Por lo tanto, los resultados observados dan soporte parcial a la hipótesis H7, en lo referido a la relación entre participación de mujeres en la propiedad y plazo del financiamiento.

- Administración y género. La participación de mujeres en el directorio no se relaciona con las variables de financiamiento (panel B), por lo cual no hay soporte para la hipótesis H9. En cuanto 
a las variables de control (panel C), no hay relaciones significativas con el sector, antigüedad, tamaño, participación del principal accionista ni con la participación de directores externos, aunque sí se observa que en las empresas familiares es mayor la participación de mujeres en el directorio. No se observan relaciones significativas con las restantes variables de GC y género (panel A), por lo cual no hay evidencia que apoye a H1 (relación entre propiedad y directorio) y H3 (relación entre directorio y alta gerencia).

Asimismo, el porcentaje de mujeres en la alta gerencia tampoco se relaciona con las variables de financiamiento (panel B), por lo cual no hay evidencia para H8. En cuanto a las variables control, el porcentaje de mujeres en la alta gerencia se relaciona positivamente con el porcentaje de directores externos $(r h o=0.4)$, pero no tiene relación con el tamaño, antigüedad, sector, empresa familiar y participación del principal accionista (panel C). Tampoco se observan relaciones significativas con las restantes variables de GC y género (panel A), lo cual deja sin evidencia a H2 (relación entre propiedad y alta gerencia).

- Auditor y género. Las empresas auditadas por mujeres tienen una tendencia a tener mayor endeudamiento con respecto al capital (panelB). La presencia de una auditora mujer no se relaciona significativamente con el sector de la empresa, tamaño medido por ventas, antigüedad, participación del principal accionista ni porcentaje de directores externos (panel C). Tampoco se observan relaciones significativas con las restantes variables de GC y género (panel A), lo que deja sin soporte a las hipótesis H4 (relación entre propiedad y auditora mujer), H5 (relación entre directorio y auditora mujer) y H6 (relación entre directorio y auditora mujer). Al no observarse relación con las variables de control, la correlación observada entre la auditora mujer y financiamiento no resulta de naturaleza espuria, por lo cual estos resultados dan soporte parcial (en cuanto a tipo de financiamiento) a la hipótesis H10.

\section{Conclusiones}

Los mecanismos de GC en pymes de países emergentes constituyen un tema poco estudiado, en especial desde una perspectiva de género. En función de los resultados de este trabajo, se establecen conclusiones en varios ámbitos. Primero, en cuanto a la relación entre GC y género, se observa que no existe relación entre la participación de mujeres en los distintos estamentos: propiedad, directorio, alta gerencia y auditor. También es interesante observar que la concentración del capital disminuye a medida que un mayor número de mujeres participan en la propiedad.

Contrariamente a lo señalado por Constantinidis et al. (2006), la tenencia accionaria en manos de mujeres se relaciona con un mayor tamaño de la empresa, resultado que podría estar explicado por la naturaleza de las empresas analizadas, que son participantes del mercado de capitales. También es interesante señalar que la participación de mujeres en la alta gerencia se relaciona positivamente con el porcentaje de directores externos, lo cual concuerda con Brunninge et al. (2007) respecto al rol que los directores externos tienen en el cambio estratégico. Asimismo, en las empresas familiares la participación de mujeres en el directorio es mayor, lo cual puede explicarse siguiendo a López Vergara et al. (2011): la motivación surgida de la conservación del patrimonio y la preservación de la unidad familiar, entre otros. Cabe destacar que las características de GC y género no presentan diferencias significativas en cuanto al sector y a la antigüedad de la empresa.

En línea con Carter et al. (2007), se observan diferencias en los perfiles de financiamiento en función de la participación de mujeres en la propiedad de la empresa. Cuando la principal accionista es mujer, existe un mayor endeudamiento a corto plazo. No se encuentra relación 
entre la participación de mujeres en el directorio, y en la alta gerencia, con el nivel y plazo de endeudamiento, resultados contrarios a lo observado por Mínguez-Vera y López-Martínez (2010). La ausencia de resultados significativos puede deberse a una relación débil entre las variables, que no resulta detectable dado el tamaño reducido de la muestra.

Existe una tendencia en las empresas auditadas por mujeres a tener mayor proporción de pasivos totales sobre el total de su patrimonio neto. Este resultado se alinea con lo observado por Ittonen et al. (2013), ya que la precisión y la eficiencia de las mujeres auditoras impactaría en una mayor confianza generada por los reportes financieros, atenuando las asimetrías informativas, y por lo tanto se asociaría con mayor endeudamiento.

Cabe mencionar que al tratarse de empresas emisoras de obligaciones negociables, las conclusiones de este estudio no son directamente extensibles a todo el universo de pymes, donde predomina el financiamiento bancario. Asimismo, el reducido tamaño de la población bajo estudio imposibilita la realización de análisis multivariados de la problemática. Las potenciales distorsiones existentes en la información contable analizada, en particular respecto a los valores del Patrimonio Neto de las empresas ${ }^{8}$, surgen también como una limitación de este estudio.

La originalidad del este trabajo reside en al menos tres aspectos. En primero lugar, se estudian los aspectos de GC y género en pequeñas y medianas empresas, cuando la gran mayoría de los antecedentes sobre este tema se han tratado en las grandes empresas. En segundo lugar, se realiza un aporte aplicado a países emergentes, para los cuales existen escasos estudios empíricos previos. Por último, se ha generado una base de datos única con la información financiera y de GC sistematizada de las pymes emisoras. Esto permite delinear como futura línea de investigación el análisis de las ventajas y desventajas de un mayor requerimiento normativo de la información relacionada con el GC para este tipo de empresas.

\section{Anexo.}

En la tabla A.1 se presentan los niveles de ventas máximas anuales (en dólares estadounidenses) para entrar en la categoría «pequeña o mediana empresa», establecidos por la Secretaría de la Pequeña y Mediana Empresa y Desarrollo Regional en su Resolución 50/13.

Tabla A.1

Definición de pyme de la Secretaría de la Pequeña y Mediana Empresa y Desarrollo Regional

\begin{tabular}{llllc}
\hline \multicolumn{5}{c}{ Sector } \\
\hline Agropecuario & Industria y minería & Comercio & Servicios & Construcción \\
\hline $6,382,978.72$ & $21,631,205.7$ & $29,550,827.4$ & $7,446,808.51$ & $9,929,078.01$ \\
\hline
\end{tabular}

Nota: valores en dólares estadounidenses, convertidos según dólar minorista vendedor Banco Nación al 16/12/2014. Fuente: elaboración propia en base a la tabla existente en la Resolución 50/13 de la Secretaría de la Pequeña y Mediana Empresa y Desarrollo Regional.

\footnotetext{
${ }^{8}$ Los estados financieros presentados en la Argentina no se encuentran corregidos por inflación. La inflación acumulada en el periodo junio 2011-mayo 2013 en la Argentina ronda el 118\% (fuente: Índice de Precios al Consumidor, Instituto de Estadística de San Luis). La Norma Internacional de Contabilidad N. ${ }^{2}$, aplicable a la información contable en economías hiperinflacionarias, plantea, entre otros aspectos, la necesidad de corrección por inflación si la tasa acumulada de inflación de 3 años se acerca o supera el $100 \%$.
} 
Tabla A.2

Listado de empresas analizadas

\begin{tabular}{|c|c|c|c|c|}
\hline Emisora & Sector & Fecha prospecto & Ventas $2013^{\mathrm{a}}$ & $\begin{array}{l}\text { Antigüedad a } \\
\text { fecha prospecto }\end{array}$ \\
\hline Cosas Nuestras SA & Comercio & Enero 2011 & $25,401,070.05$ & 8.8 \\
\hline Ersa Urbano SA & Servicios & Junio 2011 & $117,267,752.15$ & 10.4 \\
\hline Alvarez Hermanos SA & Agropecuario & Julio 2011 & $8,884,689.78$ & 50.8 \\
\hline Rizobacter Argentina SA & Agropecuario & Septiembre 2011 & $108,959,698.55$ & 28.0 \\
\hline Edisur SA & Construcción & Octubre 2011 & $13,162,900.54$ & 7.5 \\
\hline Construir SA & Construcción & Mayo 2012 & $14,891,713.19$ & 20.8 \\
\hline Ovoprot SA & Industria y minería & Septiembre 2011 & $32,159,159.22$ & 10.6 \\
\hline Asahi Motors SA & Comercio & Agosto 2012 & $47,482,719.41$ & 15.7 \\
\hline Percomin SA & Comercio & Septiembre 2011 & $12,215,918.96$ & 55.2 \\
\hline Ultracongelados Rosario SA & Industria y minería & Septiembre 2011 & $10,326,277.44$ & 13.4 \\
\hline Gama SA clase A & Servicios & Noviembre 2012 & $28,101,869.76$ & 1.0 \\
\hline Euro SA & Agropecuario & Diciembre 2012 & $17,794,658.66$ & 13.2 \\
\hline Ebucar SA & Servicios & Enero 2013 & $3,809,258.17$ & 9.9 \\
\hline Centro Card SA & Servicios & Marzo 2013 & $13,372,319.74$ & 10.6 \\
\hline AMS Food International SA & Industria y minería & Abril 2013 & $952,967.08$ & 12.0 \\
\hline Compañía Integral de Alimentos & Servicios & Diciembre 2012 & $21,033,020.54$ & 33.1 \\
\hline Big Bloom SA & Comercio & Junio 2013 & $44,730,431.50$ & 12.0 \\
\hline Meranol SA & Industria y minería & Septiembre 2011 & $51,636,784.74$ & 51.6 \\
\hline Regional Trade SA & Comercio & Junio 2013 & $28,705,700.84$ & 23.6 \\
\hline Ingacot SA & Agropecuario & Julio 2013 & $1,864,228.97$ & 11.9 \\
\hline San Juan Inversiones & Servicios & Diciembre 2012 & $514,907.44$ & 8.5 \\
\hline BF Argentina SA & Servicios & Agosto 2013 & $11,155,643.05$ & 15.3 \\
\hline Decreditos SA & Servicios & Septiembre 2013 & $18,753,485.82$ & 13.1 \\
\hline Indo SA & Servicios & Diciembre 2012 & $3,430,718.51$ & 8.0 \\
\hline Alianza Semillas SA & Industria y minería & Octubre 2013 & $21,149,003.84$ & 6.5 \\
\hline InsuAgro SA & Industria y minería & Abril 2013 & $32,936,313.33$ & 11.0 \\
\hline Empresur SA & Servicios & Noviembre 2013 & $10,428,811.66$ & 8.6 \\
\hline Savant Pharm SA & Industria y minería & Julio 2013 & $49,095,762.76$ & 19.5 \\
\hline South Management SA & Servicios & Julio 2013 & $148,419,747.31$ & 24.1 \\
\hline Actual SA & Servicios & Diciembre 2013 & $10,574,173.57$ & 23.2 \\
\hline Plasticos DISE SA & Industria y minería & Junio 2013 & $16,067,820.93$ & 24.5 \\
\hline Trantor SA & Comercio & Enero 2014 & $2,651,186.78$ & 14.8 \\
\hline Tinuviel SA & Servicios & Abril 2014 & $2,867,960.86$ & 9.3 \\
\hline
\end{tabular}

\footnotetext{
a Valores en dólares estadounidenses, convertidos según dólar minorista vendedor Banco Nación promedio para el año
} 2013 (US\$ = 5.467).

Fuente: elaboración propia con base a información de estados financieros y prospectos de emisión.

\section{Referencias}

Abor, J. y Adjasi, C. K. (2007). Corporate governance and the small and medium enterprises sector: Theory and implications. Corporate Governance, 7(2), 111-122. http://dx.doi.org/10.1108/14720700710739769

Agrestri, A. (2002). Categorical Data Analysis (2nd ed.). Wiley. DOI: http://link.springer.com/referenceworkentry/ $10.1007 \%$ 2F978-3-642-04898-2_161

Arosa, B., Iturralde, T. y Maseda, A. (2013). The board structure and firm performance in SMEs: Evidence form Spain. Investigaciones Europeas de Dirección y Economía de la Empresa, 19, 127-135. http://dx.doi.org/10.1016/j.iedee. 2012.12 .003

Banco Mundial (2010). Resultados de la World Enterprise Surveys [consultado 7 Jul 2014]. Disponible en: http://espanol.enterprisesurveys.org/data/exploreeconomies/2010/argentina\#gender 
Braga Alves, M. y Shastri, K. (2011). Corporate governance, valuation, and performance: Evidence fram a voluntary market reform in Brazil. Financial Management, 40, 139-157. http://dx.doi.org/10.1111/j.1755-053X.2010.01137.x

Brunninge, O., Nordqvist, M. y Wiklund, J. (2007). Corporate governance and strategic change in SMEs: The effects of ownership, board composition and top management teams. Small Business Economics, 29, $295-308$. http://dx.doi.org/10.1007/s11187-006-9021-2

Campos, C., Newell, R. y Wilson, G. (2002). Corporate governance devolps in emerging markets: Sharlholders in emerging markets show they're willing to pay a premium for good governance standards. McKinsey on Finance, Winter, 15-18.

Carter, S., Shaw, E., Lam, W. y Wilson, F. (2007). Gender, enterpreneurship, and bank lending: The criteria and processe used by bank loan officers in assessing applications. Enterpreneurship Theory and Practice, 31(3), $427-444$. http://dx.doi.org/10.1111/j.1540-6520.2007.00181.x

Casal, A. (2010). Gobierno Corporativo. Dirección, Administración y Control de Organizaciones en Forma Ética. Buenos Aires: Ed. Errepar.

Constantinidis, C., Cornet, A. y Asandei, S. (2006). Financing of women-owned ventures: The impact of gender and other owner- and firm-related variables. Venture Capital, 8(2), 133-157. http://dx.doi.org/10.1080/13691060600572557

Daily, C., Certo, T. y Dalton, D. (1999). A decade of corporate women: Some progress in the boardroom, none in the executive suit. Strategic Management Journal, 20(1), 93-99.

Dasilas, A. y Papasyriopoulos, N. (2015). Corporate governance, credit ratings and the capital structure of Greek SME and large listed firms. Small Business Economics, 45, 215-244. http://dx.doi.org/10.1007/s11187-015-9648-y

Farrell, K. A. y Hersch, P. L. (2005). Additions to corporate boards: The effect of gender. Journal of Corporate Finance, 11, 85-106. http://dx.doi.org/10.1016/j.jcorpfin.2003.12.001

Francoeur, C., Labelle, R. y Sinclair-Desgagné, B. (2008). Gender diversity in corporate governance and top management. Journal of Business Ethics, 81, 83-95. http://dx.doi.org/10.1007/s10551-007-9482-5

Garay, U. y González, M. (2008). Corporate governance and firm value: The case of Venezuela. Corporate Governance: An International Review, 10, 194-209. http://dx.doi.org/10.1111/j.1467-8683.2008.00680.x

Grant Thornton International, International Business Report [consultado 11 Feb 2016]. Disponible en http://www.gtar.com.ar/comunicados2015.html

Instituto Argentino de Mercado de Capitales (2013). Informe Annual [consultado 1 Oct 2014]. Disponible en: http://www.iamc.com.ar/resultadosCompleto/busqueda/informeanuario/

Ittonen, K., Vähämaa, E. y Vähämaa, S. (2013). Female auditors and accruals quality. Accounting Horizons, 27, $205-228$. http://dx.doi.org/10.2308/acch-50400

Jensen, M. y Meckling, W. (1976). Theory of the firm: Managerial behavior, agency costs, and ownership structure. Journal of Financial Economics, (3), 305-360. http://dx.doi.org/10.1016/0304-405X(76)90026-X

Jonhsen, G. y McMahon, R. (2005). Owner-manager gender, financial performance and business growth amongst SMEs from Australia's Business Longitudinal Survey. International Small Business Journal, 23(2), $115-142$. http://dx.doi.org/10.1177/0266242605050509

Kang, E., Ding, D. K. y Charoenwong, C. (2010). Investor reaction to women directors. Journal of Business Research, 63, 888-894. http://dx.doi.org/10.1016/j.jbusres.2009.06.008

Klapper, F. y Love, I. (2004). Corporate governance, investor protection, and performance in emerging markets. Journal of Corporate Finance, 10, 703-728. http://dx.doi.org/10.1016/S0929-1199(03)00046-4

López Vergara, M., Gómez-Betancourt, G. y Betancourt Ramírez, J. (2011). Factores que influyen en la participación de la mujer en cargos directivos y órganos de gobierno de la empresa familiar colombiana. Cuadernos de Administración, 24(42), 253-274.

Mínguez-Vera, A. y López-Martínez, R. (2010). Female directors and SMES: An empirical analysis. Journal of Global Strategic Management, 8, 34-46. http://dx.doi.org/10.20460/JGSM.2010415823

O'Donnell, E. y Johnson, E. N. (2001). The effects of auditor gender and task complexity on information processing efficiency. International Journal of Auditing, 5, 91-105. http://dx.doi.org/10.1111/j.1099-1123.2001.00328.x

Santolíquido D., Briozzo A., Albanese D. (2014). Gobierno corporativo, rentabilidad y financiamiento en Pymes. XXXIV Jornadas de la Sociedad de Docentes en Administración Financiera, Vaquerías, Argentina.

Young, M. N., Peng, M. W., Ahlstrom, D., Bruton, G. D. y Jiang, Y. (2008). Corporate governance in emerging economies. Journal of Management Studies, (45), 196-220. http://dx.doi.org/10.1111/j.1467-6486.2007.00752.x 